\title{
DE LA INTIMIDAD TERRITORIAL A LA INFORMATIVA: LA DEFENSA DE LA INTIMIDAD A TRAVÉS DE SUS MANIFESTACIONES CONSTITUCIONALES
}

\author{
Juan José LÓPEZ ORTEGA \\ Magistrado de la Audiencia Provincial de Madrid \\ Profesor Asociado de Derecho Procesal \\ Universidad Carlos III de Madrid \\ jjlorteg@der-pu.uc3m.es \\ Juan Manuel Alcoceba GIL \\ Profesor Ayudante Doctor de Derecho Procesal \\ Universidad Carlos III de Madrid \\ jalcoceb@der-pu.uc3m.es
}

\begin{abstract}
RESUMEN
La intimidad es quizá el derecho fundamental afectado con más habitualidad por el proceso penal contemporáneo. Sin embargo, el carácter cambiante de su contenido, solo precisable en relación con el marco bistórico y social en el que se invoca, dificulta su salvaguarda. Es por ello que, para dar protección procesal a este bien jurídico, se hace necesario articular una pluralidad de garantías capaces de cubrir las distintas facetas en las que el derecho en abstracto acaba por concretarse. Así, desde el nacimiento del Derecho moderno hasta la actualidad, la configuración jurídica de la intimidad ha ido evolucionando e incorporando a su naturaleza distintas dimensiones que, a su vez, exigen mecanismos de defensa especificos fuera y dentro del proceso. Esta exigencia se agudiza críticamente con el auge de las tecnologías de la comunicación.

Palabras clave: proceso penal, derechos fundamentales, intimidad, art. $18 \mathrm{CE}$, garantías procesales, big data, autodeterminación informativa, anonimato.

\section{ABSTRACT}

Privacy is probably the basic right which is the most affected by current criminal procedure. However, the changeable nature of its content, only established by its related historic and social background, turns its protection into a difficult matter. Therefore, in order to protect this legal being we need to plan certain guarantees so as to cover all areas in which abstract laws tend to convey. Thus, from the dawn of Modern Law to current date, the way privacy has been studied by law has evolved and added several dimensions to its nature, which also requires specific protection
\end{abstract}


J. J. López Ortega y J. M. Alcoceba Gil De la intimidad territorial a la informativa...

guarantees on and off the legal system. Such requirements become clearer with the new high tech era.

Keywords: criminal procedure, basic rights, privacy, article 18 from spanish constitution, procedural guarantees, big data, self-determination and the right to be informed, anonymity.

\section{ZUSAMMENFASSUNG}

Privatsphäre stellt vielleicht jenes Grundrecht dar, das von den derzeitigen Strafverfabren am meisten in Mitleidenschaft gezogen wird. Jedoch erschwert die wechselartige Beschaffenheit seines Inhalts, der sich nur im historischen und sozialen Zusammenhang begründen lässt, den Datenschutz. Daher müssen, um dieses Rechtsgut zu schützen, eine Vielzahl von Gewährleistungen formuliert werden, die in der Lage sind, die unterschiedlichen Facetten abzudecken, in die binein sich das abstrakte Gesetz konkretisiert. So hat sich die juristische Erfassung der Privatsphäre von der Entstebung des modernen Rechts bis heute weiterentwickelt und hat zu ibrem Wesen verschiedene Dimensionen hinzugefügt, welche ibrerseits spezifische Verteidigungsmechanismen innerbalb und außerbalb des Strafrechtsverfabrens erforderlich machen. Dieses Erfordernis spitzt sich angesichts des Aufkommens neuer Kommunikationstechnologien zu.

Schlüsselwörter: strafrechtsverfahren, grundrechte, privatsphäre, art. $18 \mathrm{Eg}$, gerichtsverfahrensgewährleistungen, big data, selbstbestimmung in der informationsgesellschaft, anonymität.

SUMARIO: I. INTRODUCCIÓN.-II. ESPECIFICIDADES DE LA INTIMIDAD COMO BIEN JURÍDICO PROTEGIDO.-III. DE LA INTIMIDAD TERRITORIAL A LA INFORMATIVA.-IV. EL DERECHO A LA AUTODETERMINACIÓN COMO GARANTÍA DE LA INTIMIDAD INFORMATIVA.-V. UN FUTURO SIN INTIMIDAD O UNA INTIMIDAD SIN FUTURO.--VI. BIBLIOGRAFÍA.

\section{INTRODUCCIÓN}

Pocas dudas caben sobre el papel central que la intimidad ocupa en el diseño del actual sistema de enjuiciamiento criminal. Su salvaguarda, así como las condiciones en que el ordenamiento jurídico permite su afectación, son cuestiones de primer orden para el legislador, que además han resultado objeto de desarrollo por parte de una extensísima jurisprudencia tanto interna como internacional. No obstante, determinar el contenido que este derecho fundamental adquiere en sus diferentes dimensiones $\mathrm{y}$, consecuentemente, definir las garantías que deben operar sobre cada una de ellas, no resulta sencillo, existiendo una pluralidad de teorías y perspectivas distintas desde las que abordar tales cuestiones. 


\section{J. J. López Ortega y J. M. Alcoceba Gil De la intimidad territorial a la informativa...}

La difícil concreción y protección del bien protegido por el art. 18 CE encuentra explicación en el carácter cambiante de la propia idea de intimidad, siempre vinculada al contexto cultural e histórico en que se invoca. Por esta razón, su positivización da lugar a una construcción jurídica cuya articulación resulta especialmente sensible a las transformaciones sociales, de entre las que destacan aquellas acontecidas al hilo de la revolución tecnológica, en tanto fenómeno característico de nuestro tiempo.

En este sentido, puede distinguirse la existencia de una concepción pretecnológica de la intimidad, basada en la potestad de excluir al conocimiento de terceros ciertos ámbitos reservados de nuestra vida o cuerpo, y una postecnológica, formulada en clave activa y no meramente defensiva, al atribuir al titular del derecho facultades para determinar el uso que se hace de la información personal que le concierne más allá del momento en el que esta se obtiene. Ambas manifestaciones del derecho fundamental son complementarias, correspondiendo la primera a su dimensión territorial y la segunda a la informativa.

Con el desarrollo exponencial de las tecnologías de la comunicación, la intimidad informativa ha ido ganando importancia progresivamente, a la par que se multiplicaban las formas de injerencia en el contenido de la misma. Así, a la inviolabilidad del cuerpo, el domicilio o las comunicaciones como mecanismos de defensa de la intimidad territorial, se suma desde finales del pasado siglo la potestad del ciudadano para controlar el uso de sus datos personales por parte de terceros a través del reconocimiento de su derecho a la autodeterminación informativa. Este derecho, que opera como cimiento axiológico sobre el que se asienta la disciplina conocida como «protección de datos», pese a ser concebido como independiente y autónomo por la jurisprudencia constitucional, ha definido la principal línea de defensa de la intimidad informativa desarrollada hasta la fecha.

Sin embargo, en el marco de una sociedad completamente interconectada y computerizada como la actual, donde la obtención masiva y posterior tratamiento automatizado de la información se presentan como una fuente inagotable de conocimiento - ya no solo sobre individuos concretos o hechos presentes y futuros, sino también sobre los fenómenos y procesos sociales que han sucedido, están sucediendo o sucederán-, cabe preguntarse si la consolidación y perfección de los tradicionales mecanismos de tutela del derecho a la intimidad, articulados en torno a la idea de autonomía individual, serán suficientes para garantizar su existencia en un futuro inmediato, o si, por el contrario, resultará necesario establecer nuevas herramientas destinadas a proteger un concepto de intimidad ya no 
solo predicable de personas o situaciones concretas, sino también de colectivos o prácticas sociales vinculadas al uso de la tecnología.

\section{ESPECIFICIDADES DE LA INTIMIDAD COMO BIEN JURÍDICO PROTEGIDO}

La intimidad es un bien jurídico que la Constitución Española ha elevado a la categoría de derecho fundamental, vinculándolo al libre desenvolvimiento de la personalidad. Respecto del mismo cabe señalar que en general se configura bajo la forma de un poder jurídico de la persona sobre un ámbito determinado, pero, más allá de este extremo, resulta difícil proporcionar una definición precisa y unívoca de la noción de intimidad ${ }^{1}$. Así lo demuestra el hecho de que se haya aludido a la misma como una «définition introuvable» ${ }^{2}$ y que por ello, en la mayoría de los casos, las legislaciones se limiten a concretar los supuestos de lesión, como el ordenamiento español hace en los arts. 18 CE y 7 de la Ley Orgánica 1/1982. Precisamente porque el contenido de este derecho fundamental presenta unos rasgos imprecisos, el Tribunal Constitucional lo ha definido desde el origen como una realidad intangible - un concepto multiforme y variable - cuya extensión ha de determinarse atendiendo a las ideas y convicciones más generalizadas en la sociedad y en cada momento histórico (STC 171/1990). La manifestación más rotunda de esta concepción de la intimidad se encuentra, probablemente, en el art. 2.1 de la Ley Orgánica 1/1982, de 5 de mayo, de Protección Civil del Derecho al Honor, a la Intimidad Personal y Familiar y a la Propia Imagen, que apela a los usos sociales como categoría delimitadora de la misma.

Pero la condición poliédrica del concepto jurídico de intimidad no hace sino evidenciar su pertenencia al acervo de los derechos humanos, habida cuenta de que la historicidad es uno de los rasgos más característicos de este tipo de derechos, que no se conciben como categorías cerradas y definitivas, sino en constante evolución ${ }^{3}$. Ello es así dado que, como

${ }^{1}$ J. J. López Ortega, «La intimidad como bien jurídico protegido», en T. S. Vives Antón (dir.), Estudios sobre el Código Penal de 1995 (Parte Especial), Madrid, Consejo General del Poder Judicial, 1996, pp. 283-310.

2 A. Vitalis, Informatique, Pouvoir et Libertés, Paris, Economica, 1981.

3 Sobre este extremo puede consultarse G. Peces-Barba MarTínez, «Sobre el fundamento de los derechos humanos: un problema de moral y derecho», en J. MugueRza y G. Peces-Barba Martínez (eds.), El fundamento de los derechos humanos, Madrid, Debate, 1989, pp. 265-277, disponible en bttp://bdl.handle.net/10016/12917. 


\section{J. J. López Ortega y J. M. Alcoceba Gil De la intimidad territorial a la informativa...}

cualquier obra de la actividad humana, son producto de su tiempo, y solo pueden comprenderse en un contexto cultural y temporalmente determinado $^{4}$. De hecho, es ya un lugar común para la doctrina distinguir tres diferentes generaciones sucesivas de derechos: las libertades públicas; los derechos económicos, sociales y culturales, y, en la sociedad actual, los surgidos para contrarrestar la corrupción de las libertades provocada por las consecuencias indeseadas de la revolución tecnológica ${ }^{5}$. En esta última categoría es donde habrían de ubicarse las expresiones del derecho a la intimidad en su vertiente informativa: la autodeterminación informativa, la protección de datos, el anonimato digital o el derecho al olvido ${ }^{6}$.

\section{DE LA INTIMIDAD TERRITORIAL A LA INFORMATIVA}

La aparición de la intimidad en la esfera jurídica se ha vinculado tradicionalmente con la emergencia de la burguesía como clase social dominante en torno a la cual pivota el concepto moderno de privacy ${ }^{7}$. Concebida en su origen como derecho a la soledad y al aislamiento, al inicio se concretaba en el reconocimiento de ciertas facultades dirigidas a salvaguardar un espacio de carácter exclusivo y excluyente; notas que recuerdan la delimitación y defensa del derecho de propiedad en el que se fundamentaba

${ }^{4}$ Si tal y como señala G. Peces-Barba Martínez, ibid., «sin organización económica capitalista, sin cultura secularizada, individualista y racionalista, sin el Estado soberano moderno que pretende el monopolio en el uso de la fuerza legítima, sin la idea de un Derecho abstracto y de unos derechos subjetivos, no es posible plantear esos problemas de la dignidad del hombre, de su libertad e igualdad desde la idea de los derechos humanos», lo mismo sucedería con la sociedad de la información, Internet, las bases de datos y derechos como la autodeterminación y la intimidad informativa, la libertad informática o la tutela de los datos personales.

${ }_{5}$ De forma similar, A. E. PéREZ LuÑo, «Las nuevas tecnologías y la garantía procesal de las libertades», Revista de Derecho de la UNED, núm. 11 (2012), pp. 1007-1020, señala cómo «en nuestro tiempo ha aparecido una nueva o "tercera generación" de derechos humanos. Estos nuevos derechos se refieren a cuestiones tales como el derecho a la paz, los derechos de los consumidores, el derecho a la calidad de vida y a la protección del medio ambiente, el derecho al desarrollo, la libertad informática con la consiguiente tutela de los datos personales, las garantías frente a determinados usos de las biotecnologías...».

${ }^{6}$ En el mismo sentido señala A. E. PÉREZ LuÑo, «Del habeas corpus al habeas data», Informática y derecho: Revista iberoamericana de derecho informático, núm. 1 (1992), pp. 153 161, como «aludir a un tránsito desde el habeas corpus al habeas data representa aceptar, de forma implícita, que existe una evolución de los derechos y libertades, o, si se prefiere, que estamos asistiendo a un proceso evolutivo de generaciones de derechos humanos».

${ }^{7}$ Sobre el origen jurídico del derecho a la intimidad vid. L. Rebollo Delgado e Y. Gómez SÁncheZ, Biomedicina y protección de datos, Madrid, Dykinson, 2008, pp. 23 y ss. 


\section{J. J. López Ortega y J. M. Alcoceba Gil De la intimidad territorial a la informativa...}

la noción jurídica primigenia de intimidad ${ }^{8}$. La doble concreción del right to be let alone - en las ideas de soledad y tranquilidad - marca el contexto en que se sentaron las primeras bases técnico-jurídicas de la intimidad, entendida como garantía de los individuos frente a cualquier intromisión en el recinto de su esfera privada o como derecho del individuo a una vida retirada y anónima9. Cabe destacar que, debido al estado de la técnica en aquel momento, obstaculizar el uso de los sentidos — la vista o el oídomediante impedimentos físicos como muros o cortinas bastaba para preservar su intimidad, por lo que la tutela del derecho se centraba en impedir o castigar las intromisiones indeseadas en los espacios objeto de protección, configurándolo como una facultad negativa de carácter defensivo. Sin embargo, la emergencia de los derechos humanos como respuesta a los totalitarismos de la primera mitad del siglo xx provocará la primera transformación del contenido del derecho, que se separa de la idea de propiedad para fundamentarse en la dignidad humana, en la inviolabilidad de la persona y, en último término, en la idea de libertad. Así, el valor dignidad humana, como fundamento de la intimidad, incorpora la afirmación positiva del libre desarrollo de la personalidad del individuo, que se concreta en su total autodeterminación ${ }^{10}$.

La dignidad de la persona se convierte, de este modo, en el elemento fundamental para calibrar el alcance y el significado del derecho a la intimidad, que atiende, sobre todo, al desarrollo de la propia individualidad. Por tanto, la intimidad pasa a ser definida, a partir del giro descrito, como defensa frente a la publicidad y, por ende, como capacidad de control de las informaciones que sobre uno mismo pueden tener las personas ajenas ${ }^{11}$, lo que lleva a la limitación de los mecanismos de control social o estatal basados en el acopio indiscriminado y secreto de información sobre sus ciudadanos. Por esta razón, y ante el gran desarrollo experimentado por las tecnologías, la universalización de la informática, Internet y la consiguiente sofisticación de los sistemas de vigilancia, comienza a resultar insuficiente la concepción de la intimidad como mero medio de defensa frente a las invasiones indebidas en la esfera privada, haciéndose necesario el desarrollo de una nueva

${ }^{8}$ S. D. Warren y L. D. Brandesis, «The Right to the Privacy», Harvard Law Review, vol. 4, núm. 5 (1890), pp. 193-219.

9 W. L. Prosser, «Privacy», California Law Review, núm. 48 (1960), pp. 383-423.

${ }_{10}$ A este respecto puede consultarse P. L. MuRillo de LA Cueva, El derecho a la autodeterminación informativa, Madrid, Tecnos, 1990.

11 A. F. Westin, «Privacy And Freedom», Wash. E Lee L. Rev, vol. 25, núm. 166 (1968), pp. 166-170, disponible en bttp://scholarlycommons.law.wlu.edu/wlulr/vol25/iss1/20. 
dimensión del derecho, esta vez en clave activa, capaz de otorgar a su titular el control sobre el flujo de informaciones que le afectan ${ }^{12}$.

En efecto, si en su origen la intimidad era concebida como una típica libertad de signo individual, circunscrita al ámbito del hombre aislado, es decir, como un derecho a la soledad, a la reserva y al aislamiento, que se concretaba en el reconocimiento de unas facultades dirigidas a preservar un espacio exclusivo y excluyente, durante la segunda mitad del pasado siglo, el centro de gravedad en la delimitación conceptual de este derecho se desplaza hacia el poder de control sobre las informaciones que son relevantes para cada sujeto, surgiendo así una creciente preocupación por la dimensión informativa de la intimidad, cuya existencia solo resulta explicable desde una concepción intersubjetiva social o externa del Derecho.

No obstante, será a finales de la década de los noventa cuando se produzcan los mayores avances en el reconocimiento del contenido informacional de la intimidad. Hasta entonces, el derecho se configura como una realidad diferenciada de la protección del babeas data, lo que hará beneficiaria a la intimidad de una protección más intensa que la recibida por los meros datos personales. Ello sobre la base de estar dotada de un contenido material (privacy) que se identifica con el secreto, es decir, con la idea de «ámbito reservado» vinculado a la tutela de realidades físicas. De esta forma, hasta prácticamente el nuevo siglo, sus mecanismos de protección permanecerán centrados en la preservación de los ámbitos espaciales íntimos, como son, fundamentalmente, el domicilio (art. 18.2 CE), el espacio íntimo por excelencia en la configuración liberal del derecho a la intimidad, conectada con la protección del derecho de propiedad; pero también los procesos comunicativos que cada uno establece con los demás (art. 18.3 CE), los papeles reservados (art. 197.1 CP) y la propia realidad corporal (STC 37/1989).

En todos estos ámbitos siempre hay un elemento territorial. Son ámbitos que el sujeto quiere mantener resguardados del conocimiento ajeno y, por ello, la intromisión se consuma por el mero acceso al espacio físico, independientemente del conocimiento que se llegue a obtener como consecuencia de la intromisión. Sin embargo, a partir de los años 2000, la evolución experimentada por la configuración tradicional de la intimidad llevará a extender la protección a nuevos lugares, es decir, a descubrir nuevos ámbitos de intimidad territorial, como, por ejemplo, sucede con las celdas

12 A. E. Pérez LuÑo, «Perfiles morales y políticos del derecho a la intimidad», Anales de la Real Academia de Ciencias Morales y Políticas, núm. 73 (1996), pp. 311-340. 


\section{J. J. López Ortega y J. M. Alcoceba Gil De la intimidad territorial a la informativa...}

de los establecimientos penitenciarios (STC 89/2006), en las que opera la protección conferida por el art. 18.2 CE a pesar de no ser domicilio; los equipajes (STC 281/2006); el buzón en el que se recibe la correspondencia (ATC 104/2000); el ordenador personal (STC 173/2011), y el terminal de telefonía (STC 142/2012) ${ }^{13}$.

Todas ellas son realidades materiales, contextos espaciales protegidos por una dimensión de intimidad definible como territorial. Pero si se reconoce a su titular la facultad de excluirlos del conocimiento ajeno es, sobre todo, por las informaciones que pueden incorporar. Este será el aspecto más destacado en la evolución de la noción jurídica de intimidad que se desarrolla a partir de la STC 207/1996. No solo se configuran ámbitos de intimidad distintos a los manejados hasta esa fecha, sino que al combinar lo territorial con lo informativo se produce un cambio sustancial. Se dota al derecho a la intimidad de un contenido positivo y, al hacerlo, se incorpora al contenido del derecho el haz de facultades de control sobre la información que hasta ese momento quedaban fuera de la esfera de protección del mismo, lo que permite conectar la autodeterminación informativa (art. 18.4 CE) y la protección de la intimidad personal (art. 18.1 CE) ${ }^{14}$.

\section{EL DERECHO A LA AUTODETERMINACIÓN COMO GARANTÍA DE LA INTIMIDAD INFORMATIVA ${ }^{15}$}

La primera referencia al tratamiento de la información como elemento nuclear del derecho a la intimidad se encuentra en la Sentencia de 15 de diciembre de 1983 dictada por el Tribunal Constitucional Federal de Alemania (TCFA) en relación con la ley del censo ${ }^{16}$. La sentencia declaró la inconstitucionalidad de dicha norma en virtud de la excesiva cantidad de información personal que se requería al ciudadano para, con posterioridad, someterla a tratamiento informatizado. Concretamente, el Alto Tribunal alemán entendió que el contenido de la ley resultaba incompatible con

13 Sobre este último aspecto puede consultarse J. J. López OrTEgA (dir.), Derecho a la intimidad: nuevos y viejos debates, Madrid, Dykinson, 2017.

${ }_{14}$ En la misma línea se encuentra la STS 144/1999.

15 Para mayor información sobre la relación existente entre autodeterminación informativa e intimidad puede consultarse J. M. AlCOCEBA GIL, El análisis genético forense en el proceso penal español, Valencia, Tirant lo Blanch, 2018.

${ }_{16}$ Para un estudio en profundidad de la citada resolución puede consultarse D. DARANAS, «Sentencia de 15 de diciembre de 1983: Ley del Censo. Derecho de la personalidad y dignidad humana», Boletín de Jurisprudencia Constitucional, núm. 3 (1984). 
la autodeterminación informativa, en tanto derecho que proscribe la promulgación de normas en virtud de las cuales el ciudadano «no pueda percibir con suficiente seguridad qué informaciones relativas a él son conocidas en determinados sectores de su entorno social» ${ }^{17}$.

Mediante tal resolución, el TCFA vincula el derecho a la intimidad informativa con las libertades públicas, al expresar cómo la posibilidad de conocer y controlar la información personal a la que los demás tienen acceso es aquello que posibilita que las personas adquieran capacidad de decisión sobre sus actos, pues solo teniendo constancia de las repercusiones informativas de estos podrán tomarse tales decisiones libremente ${ }^{18}$. La consecuencia de este razonamiento, según ciertos sectores doctrinales, ha sido el establecimiento por parte de la jurisprudencia de un derecho fundamental a la autodeterminación informativa, construido con base en el derecho general de la personalidad y cuyo ámbito de protección se centra en «la recogida, almacenamiento y utilización y la transmisión ilimitada de los datos de carácter personal, con el fin de garantizar la facultad del individuo de decidir libremente y por sí mismo acerca del uso y difusión de sus datos personales» ${ }^{19}$.

En diferente sentido, otros autores han indicado cómo la citada resolución judicial fue la impulsora del reconocimiento, que posteriormente se generalizaría, de la información como elemento esencial del derecho a la intimidad, al admitir expresamente que «el interesado posee la autodeterminación informativa como una facultad para el resguardo del derecho a la intimidad, haciendo especial hincapié en la necesidad de evitar la elaboración de un perfil de la persona a partir de la interacción de archivos que guardan distintos datos personales del individuo» ${ }^{20}$.

${ }^{17}$ Ibid

18 A este respecto manifiesta la citada sentencia cómo «la autodeterminación del individuo presupone - también en las condiciones de las técnicas modernas de tratamiento de la información- que se conceda al individuo la libertad de decisión sobre las acciones que vaya a realizar, o, en su caso, a omitir, incluyendo la posibilidad de obrar de hecho en forma consecuente con la decisión adoptada. Esta libertad de decisión, de control, supone además que el individuo tenga la posibilidad de acceder a sus datos personales, que pueda no solo tener conocimiento de que otros procesan informaciones relativas a su persona, sino también someter el uso de estas a un control, ya que, de lo contrario, se limitará su libertad de decidir por autodeterminación».

19 R. Martínez Martínez, «El derecho fundamental a la protección de datos: perspectivas», Revista de Internet de Derecho y Política, núm. 5 (2007), disponible en bttp://www. uoc.edu/idp/5/dt/esp/Martinez.pdf.

${ }^{20}$ M. GaLÁn JuÁreZ, Intimidad: nuevas dimensiones de un viejo derecho, Madrid, Centro de Estudios Ramón Areces, 2005, p. 209. 


\section{J. J. López Ortega y J. M. Alcoceba Gil De la intimidad territorial a la informativa...}

No obstante, el desarrollo jurisprudencial de este derecho se ha asociado, por lo general, con la protección de datos de carácter personal, siendo enunciado también por el Tribunal Europeo de Derechos Humanos en relación con el denominado babeas data ${ }^{21}$. De hecho, ha sido específicamente en el momento de su traslación al ámbito nacional, que tuvo lugar una década después de la citada sentencia del TCFA, cuando se ha visto vinculado de forma directa con la tutela de la intimidad personal del art. 18.1 CE, a raíz de la interpretación que el Tribunal Constitucional español ha realizado del mismo ${ }^{22}$.

Aun así, nuestra jurisprudencia también ha mantenido la diferenciación entre las nociones de intimidad y autodeterminación informativa, tratándolas como si fuesen dos derechos diferentes, pese a que este intento de diferenciación pueda considerarse estéril y artificioso además de inútil, en la medida que de él no parece derivarse aportación alguna a la construcción dogmática del derecho a la intimidad ${ }^{23}$.

Ello es así dado que la configuración del art. $18 \mathrm{CE}$ diseñada por las SSTC 290/2000 y 292/2000 parece ignorar el hecho de que las concreciones territoriales de la intimidad también presentan un contenido informativo $^{24}$. Si se accede al cuerpo del investigado, a su domicilio o si se vigilan sus conversaciones no es más que para obtener informaciones, fuentes de prueba que se utilizan en la investigación. Por lo que, aunque en la doctrina constitucional todavía hoy sea común aceptar la autonomía de las diversas manifestaciones de la intimidad, no se puede ignorar que las distintas

${ }^{21}$ Sobre el desarrollo del derecho a la autodeterminación informativa en relación con la protección de datos puede consultarse R. MARTínEZ MARTínEZ, «El derecho fundamental a la protección de datos...», op. cit... pp. 47-61.

${ }^{22}$ Formulándose por primera vez en la STC 207/1996 en relación con el conocimiento de si el sujeto es drogadicto a través de pruebas médicas, seguidamente en la STC 243/1997 en relación con las informaciones que se pueden obtener al realizar la prueba de alcoholemia y en la STC 134/1999 en relación con la información que proporcionaba una fotografía obtenida en un lugar público por un grupo de amigos.

23 J. J. LÓPEZ ORTEGA, «La tutela de la intimidad genética en la investigación penal. A propósito de la STC 199/2013 y de la SAP Sevilla 650/2013», en M. CASADO y M. GuILléN (coords.), ADN forense: problemas éticos y jurídicos, Barcelona, Observatori de Bioetica i Dret-Universidad de Barcelona, 2014, pp. 99-119.

${ }^{24}$ A partir de la cual se otorgaba carácter autónomo al derecho a la autodeterminación informativa, consagrándolo como un nuevo derecho fundamental distinguible de la intimidad personal en tres aspectos concretos: $i$ ) el carácter defensivo de la intimidad ante el derecho a la autodeterminación como potestad; ii) el bien jurídico protegido, ya que la autodeterminación protege la capacidad del sujeto para controlar su información personal, sea o no privada, e iii) el «secreto» que comporta la intimidad y que no requiere la autodeterminación informativa. 


\section{J. J. López Ortega y J. M. Alcoceba Gil De la intimidad territorial a la informativa...}

formas de injerencia que se consagran en la Constitución no son en realidad más que singularizaciones de un mismo bien jurídico. Si la inviolabilidad del domicilio, el secreto de las comunicaciones y el derecho a la intimidad corporal reflejan una concepción territorial del derecho en cuanto circunscriben el objeto de protección a determinados espacios o zonas que se mantienen ocultos y reservados frente a la acción de los demás, la autodeterminación informativa proporciona una protección más amplia, puesto que su atención se centra en las informaciones relativas a la vida privada de las personas. Pero, en todos los casos, estamos ante manifestaciones de la intimidad, concretamente aquellas más conocidas y frecuentes en el momento en que se elaboró la Constitución, sin que pueda derivarse de ellas nada sustancialmente distinto de la propia noción que se consagra como una garantía genérica en el art. 18.1 CE. Así, la dimensión informativa no constituye una realidad autónoma e independiente del resto, sino, en realidad, una cara más de la intimidad que sirve como fundamento tanto a la autodeterminación informativa como al resto de derechos que pretenden dotar de control a su titular sobre la exposición pública de su persona al entorno tecnológico.

\section{UN FUTURO SIN INTIMIDAD O UNA INTIMIDAD SIN FUTURO}

Llegados al tiempo presente, parece clara la necesidad de volver a definir el contenido del derecho a la intimidad, integrando ahora en él los elementos necesarios para que pueda ser también objeto de protección en el contexto tecnológico donde hoy en día tiene lugar su ejercicio.

Cada vez son más las voces que señalan cómo, en el mundo actual, todos los ciudadanos, desde su nacimiento, se hallan expuestos a multitud de injerencias en su vida privada, provocadas en la mayoría de los casos por las dinámicas que en las sociedades desarrolladas rigen las relaciones intersubjetivas y de las cuales, por su carácter cotidiano, no es posible sustraerse sin incurrir en riesgo de exclusión social o marginación. Sirvan de ejemplo el uso de las redes sociales y los sistemas de comunicación en tiempo real, la banca electrónica, la utilización de dispositivos que comportan la geolocalización permanente o la práctica del denominado self tracking.

En un entorno conformado íntegramente por la tecnología como es, por ejemplo, el digital, los conceptos tradicionales de defensa de la intimi- 
dad resultan inoperantes. La autodeterminación informativa - junto con el resto de manifestaciones de este bien jurídico contempladas en la Constitución - se torna tan imprescindible en sus respectivos ámbitos (informativo y territorial) como incapaz de garantizar la supervivencia de la intimidad en los espacios tecnológicos abiertos, donde la información se transmite sin ningún control del emisor. Por eso, para garantizar su salvaguarda en dichos espacios, ha de incorporarse a la formulación del derecho una nueva manifestación del mismo adaptada al momento actual, en el que los Estados parecen poseer la capacidad de monitorizar permanentemente a sus ciudadanos a partir del rastro informacional que estos dejan tras de sí y la cesión de datos personales se ha generalizado hasta el extremo de convertirse en uno de los principales negocios existentes.

La solución más obvia a la cuestión planteada parece ser la transmutación en espacios íntegramente virtuales de la intimidad como derecho al anonimato, al olvido, o, en esencia, en la capacidad de determinar cómo, cuándo y en qué medida se genera información susceptible de ser conocida por otros, así como hasta qué momento esa información debe ser accesible para los demás. Sin embargo, tales posibilidades dependen en gran medida de la arquitectura del sistema de obtención y transmisión de dicha información, y condicionar su diseño con el fin de salvaguardar la intimidad de los usuarios solo parece posible a través de una concepción colectiva de este derecho, cuya titularidad recaería en el conjunto de ellos como grupo afectado por su uso. Tal planteamiento conecta además con el debate sobre los límites en la obtención y explotación de los metadatos, hasta ahora fuera del ámbito de la intimidad por no contener información personal, pero, a la luz de los últimos acontecimientos, capaces de poner en riesgo el ejercicio de las libertades democráticas fundamentales de las que la intimidad es tributaria.

\section{BIBLIOGRAFÍA}

Alcoceba GIL, J. M.: El análisis genético forense en el proceso penal español, Valencia, Tirant lo Blanch, 2018.

Daranas, D.: «Sentencia de 15 de diciembre de 1983: Ley del Censo. Derecho de la personalidad y dignidad humana», Boletín de Jurisprudencia Constitucional, núm. 3 (1984).

GalÁn JuÁrez, M.: Intimidad: nuevas dimensiones de un viejo derecho, Madrid, Centro de Estudios Ramón Areces, 2005. 
López Ortega, J. J.: «La intimidad como bien jurídico protegido», en T. S. Vives Antón y J. L. Manzanares Samaniego (dirs.), Estudios sobre el Código Penal de 1995 (Parte Especial), Madrid, Consejo General del Poder Judicial, 1996, pp. 283-310.

- «La tutela de la intimidad genética en la investigación penal. A propósito de la STC 199/2013 y de la SAP Sevilla 650/2013», en M. CASADO y M. GuILLÉN (coords.), ADN forense: problemas éticos y jurídicos, Barcelona, Observatori de Bioetica i Dret-Universidad de Barcelona, 2014, pp. 99-119.

- (dir.): Derecho a la intimidad: nuevos y viejos debates, Madrid, Dykinson, 2017.

Martínez Martínez, R.: «El derecho fundamental a la protección de datos: perspectivas», Revista de Internet de Derecho y Política, núm. 5 (2007), disponible en http://www.uoc.edu/idp/5/dt/esp/Martínez.pdf.

Murillo De la Cueva, P. L.: El derecho a la autodeterminación informativa, Madrid, Tecnos, 1990.

Peces-Barba Martínez, G.: «Sobre el fundamento de los derechos humanos: un problema de moral y derecho», en J. Muguerza y G. Peces-Barba MartíNEZ (eds.), El fundamento de los derechos humanos, Madrid, Debate, 1989, pp. 265-278.

Perez-Luño, A. E.: «Del Habeas Corpus al Habeas Data», Informática y derecho: Revista iberoamericana de derecho informático, núm. 1 (1992), pp. 153-161.

- «Perfiles morales y políticos del derecho a la intimidad», Anales de la Real Academia de Ciencias Morales y Políticas, núm. 73 (1996), pp. 311-340.

- «Las nuevas tecnologías y la garantía procesal de las libertades», Revista de Derecho de la UNED, núm. 11 (2012), pp. 1007-1020.

Prosser, W. L.: «Privacy», California Law Review, vol. 48, núm. 3 (1960), pp. 383-423.

Rebollo Delgado, L., y Gómez Sánchez, Y.: Biomedicina y protección de datos, Madrid, Dykinson, 2008.

Vitalis, A.: Informatique, Pouvoir et Libertés, Paris, Economica, 1981.

Warren S. D., y Brandesis, L. D.: «The Right to the Privacy», Harvard Law Review, vol. 4, núm. 5 (1890), pp. 193-220.

Westin, A. F.: «Privacy And Freedom», Wash. E Lee L. Rev., vol. 25, núm. 1 (1968), pp. 166 y ss. 